\title{
IMPORTANCIA DE LOS MAMÍFEROS SILVESTRES EN LA DIETA DE POBLADORES DE LA PENÍNSULA DE OSA, COSTA RICA
}

\author{
MARIANA ALTRICHTER \\ PRMVS, Universidad Nacional, Apdo. 1350-3000 Costa Rica \\ (correo electrónico: maltrichter@yahoo.com). \\ ${ }^{1}$ Domicilio actual: School of Renewable Natural Resources, University of Arizona, 104 \\ BioSciences East, Tucson, AZ 85721
}

Resumen. Estimé la importancia que la carne de mamíferos silvestres tiene en la dieta de pobladores de la Península de Osa, cercanos al Parque Nacional Corcovado. Visité 16 pueblos y la Comunidad Indígena Guaymí e hice entrevistas a un 55 \% de las familias. La carne de monte se consume en quinto lugar, siendo el tepezcuintle Agouti paca el mamífero silvestre más consumido, seguido por el chancho cariblanco Tayassu pecari y el saíno T. tajacu $(\mathrm{H}=128$, $\mathrm{p}<0.01)$. El tepezcuintle se consume en promedio 3 veces por año por familia y el chancho una vez cada 3 años $(U=128.5, \mathrm{p}<0.01)$. Se consume significativamente más chanchos en los pueblos que limitan con el parque, mientras que el consumo de saíno aumenta con la distancia al parque. La cacería de chanchos está concentrada en dos momentos del año, mientras que los tepezcuintles y los saínos son cazados durante todo el año. La carne de monte no es la principal fuente de proteínas en esta región. La cacería de mamíferos es altamente selectiva y responde más al sabor de la carne que a una necesidad económica. La cantidad de chanchos cazados es baja, sin embargo, los chanchos que son cazados provienen del Parque Nacional Corcovado, por lo que toda la población es susceptible al efecto de la cacería. Se debería intensificar el control de la misma en los pueblos más cercanos al PNC, especialmente durante el final de la época húmeda (octubre a diciembre).

Abstract. I evaluated the importance of wild mammals' meat in the diet of villagers of the Osa Peninsula, near to Corcovado National Park. I visited 16 towns and the Guaymí Indian Reserve. I interviewed $55 \%$ of the families of the area. Among all the consumed meats, the wild meat takes a fifth place. The agouti Agouti paca is the wild mammal more consumed, followed by the white-lipped Tayassu pecari and the collared peccary T. tajacu $(\mathrm{H}=128, \mathrm{p}<0.01)$. The agouti is consumed on average three 3 times per year per family and the white-lipped peccary once every year $(\mathrm{U}=128.5, \mathrm{p}<0.01)$. The consumption of white-lipped peccaries is significantly higher in communities closer to the park, while the consumption of collared peccaries is higher in communities distant to the park. The hunt of white-lipped peccaries occurs twice in the year, while agoutis and collared peccaries are hunted throughout the year. The wild meat is not the main source of protein in this region. People are very selective to consume wild meat. The consumption of wild meat is rather related to flavor that to economic need. The amount of white-lipped peccaries hunted by the peninsula residents is low. However, white-lipped peccaries that are actually hunted came from the Park, thus, the whole 
population is vulnerable. The control of hunting should increase in the closer towns to the park and specially at the end of the wet season (from October to December).

Palabras clave: Cacería, carne de monte, Costa Rica, mamíferos, Península de Osa, subsistencia, Tayassu pecari.

\section{INTRODUCCIÓN}

En América Latina, la cacería comercial y de subsistencia ha sido siempre un recurso importante (Robinson y Redford, 1991). La carne de monte es más consumida que la carne de animales domésticos en gran parte de la Amazonía, ya que frecuentemente es el tipo de carne más accesible (Ojasti, 1993). Por ejemplo, el chancho cariblanco (Tayassu pecari) es una de las especies de mamífero más amenazadas del neotrópico (Sowls, 1997) y es considerado una importante fuente de proteína animal para muchos grupos indígenas y rurales (Nietschman, 1972; Robinson y Redford, 1991; Smith, 1976; Sowls, 1997). Sin embargo, en muy pocas áreas de Mesoamérica se conoce realmente el lugar que la carne de ésta y de otras especies de mamíferos silvestres ocupa en la dieta de la gente que vive cerca de áreas naturales protegidas. El hecho de que haya gente viviendo en los alrededores de las áreas protegidas, haciendo uso de los recursos naturales de las mismas, es una realidad que no puede dejarse de lado al planear estrategias de conservación. La participación de la gente local en la conservación de sus propios recursos naturales ha demostrado ser una importante ayuda en acciones de conservación (Vickers, 1991). Para ello, es indispensable primero conocer el papel que la vida silvestre tiene en la vida de la gente, tanto económica como culturalmente. Los objetivos de este estudio fueron estimar la importancia de la carne de mamíferos silvestres en la dieta de pobladores cercanos al Parque Nacional Corcovado y estimar la situación del chancho cariblanco fuera del Parque.

\section{AREADEESTUDIOYMÉTODOS}

Visité todas las localidades del oeste de la Península de Osa: 16 pueblos rurales y una comunidad Indígena Guaymí. Agujita es el pueblo más grande de la región, con 52 familias. Éste y Florida son pueblos turísticos ubicados sobre la playa. Rancho Quemado y la Reserva Indígena Guaymí limitan con el Parque Nacional Corcovado (PNC), y el resto de los pueblos son del interior de la península (Fig. 1). Todos estos pueblos están dentro de la Reserva Forestal Golfo Dulce. Hice entrevistas semiestructuradas (Ander Egg, 1991) a un adulto de cada casa visitada, por lo que cada entrevista representa una familia. En los pueblos pequeños entrevisté a todas las familias presentes y en los más grandes visité al menos a un 30\% de las familias. Hice en total 153 entrevistas, representando un 55 \% de las familias de la región. Reuní los 


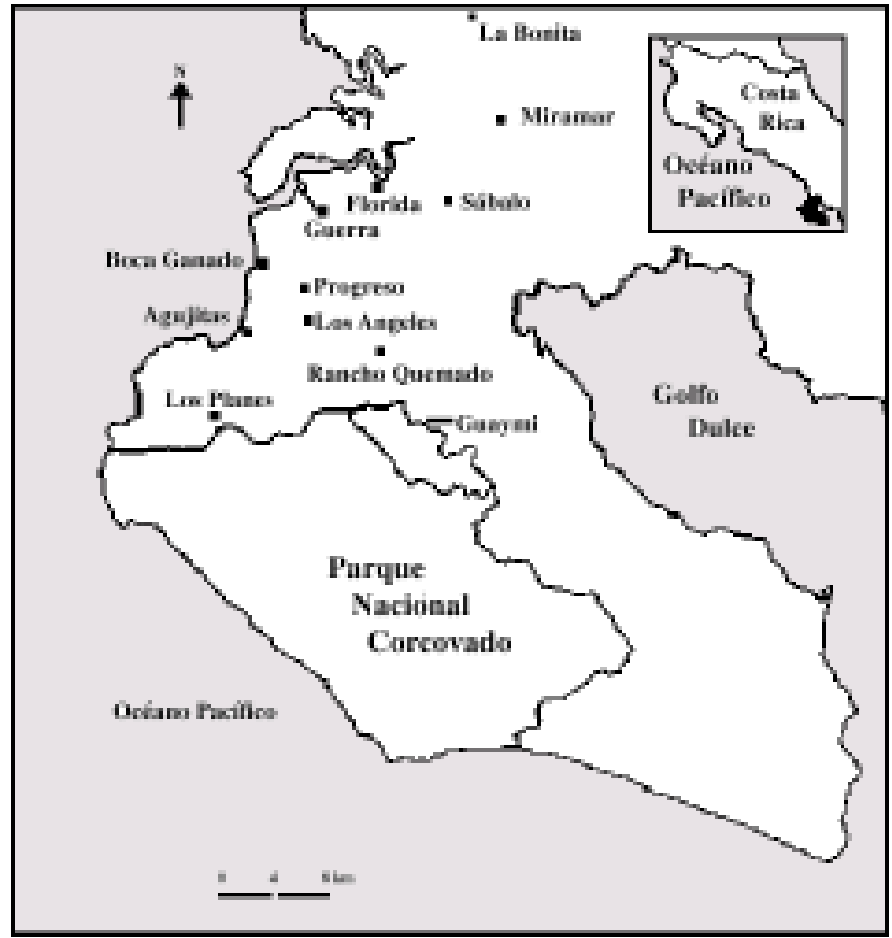

Figura 1. Area de estudio. Pueblos con más de siete familias donde se realizaron entrevistas en la Península de osa, Costa Rica.

datos de los pueblos más pequeños (menos de 10 familias) con los del pueblo mayor más cercano. Así, 12 pueblos fueron utilizados en los análisis (Fig. 1).

Para conocer el orden de importancia de consumo de carnes asigné un valor cinco a la carne más consumida, cuatro a la siguiente y así sucesivamente hasta un valor cero cuando no es consumida. Consideré un valor cinco como máximo teniendo en cuenta que las posibles carnes para consumo son cinco: res, pollo, cerdo, pescado, y carne de monte. Dentro de las carnes de monte también asigné un valor cinco a la carne más consumida. A partir de un estudio piloto determiné las siguientes especies como potenciales constituyentes de la dieta en la península: chancho de monte (Tayassu pecari), saíno (T. tajacu), tepezcuintle (Agouti paca), pizote (Nasua narica) 
y guatuza (Dasyprocta punctata). Para cada una de las carnes sumé el valor de consumo de cada familia y lo dividí por el número de familias entrevistadas obteniendo un valor medio de consumo (VMC) para el área total y un VMC por pueblo. La frecuencia de consumo de cada especie la estimé como el número de veces que una familia consume carne de esa especie por año o por mes. Estimé la frecuencia media de consumo para toda el área y la frecuencia media de consumo por pueblo. Los resultados se presentan con la media y el desvío estándar. Además hice preguntas sobre situación económica familiar, sitios de desplazamientos de chanchos, épocas de cacería, modo en que la gente consigue la carne de monte y tendencias de la fauna.

\section{RESULTADOS}

El 60\% de los entrevistados poseé fincas de entre 0.5 y 40 ha y el resto entre 40 y 530 ha. La suma total de propiedad en finca de 100 entrevistados fue de 6,671 ha. De ésto, un $65 \%$ está cubierto de bosque. La actividad principal en la región (62\%) es la agricultura de subsistencia.

La carne de monte se consume en quinto lugar $(\mathrm{VMC}=0.9$, d.e $=1.1)$, después del cerdo $(\mathrm{VMC}=4.2$, d.e $=0.9)$, el pollo $(\mathrm{VMC}=4.1$, d.e $=0.9)$, la res $(\mathrm{VMC}=3.3$, d.e $=$ 0.9 ) y el pescado $(\mathrm{VMC}=1.2$, d.e $=1.7)$. El $50 \%(\mathrm{n}=123)$ de los entrevistados compra únicamente la carne de res, y las demás carnes que consume son de animales propios o regalados. El VMC de carne de monte difiere entre pueblos $(\mathrm{H}=36.2, \mathrm{p}<0.001, \mathrm{n}=$ 149), siendo mayor en Rancho Quemado y Guaymí (colindantes con el PNC), y menor en Florida. La especie de mamífero silvestre más consumida es el tepezcuintle (VMC = 3.7, d.e = 2.8), seguida por el chancho cariblanco $(V M C=1.7$, d.e $=2.3$ ) y el saíno (VMC $=0.7$, d.e $=1.7)(\mathrm{H}=128, \mathrm{p}<0.01)$. Otros mamíferos consumidos son la guatuza y el pizote, pero en muy pequeña cantidad. El tepezcuintle se consume en promedio 3 veces por año por familia y el chancho una vez cada 3 años $(U=128.5, \mathrm{p}<0.01)$. La frecuencia de consumo de chancho fue mayor en Rancho Quemado y Guaymí que en los otros pueblos, mientras que la de saíno fue mayor en Guerra, un pueblo del interior de la península. El consumo de carne de chancho de monte es diferente entre los pueblos $(H=24.3, p<0.01)$. Se observa en general un gradiente de disminución de consumo de carne de chancho a medida que se agranda la distancia al PNC , mientras que el consumo de carne de saíno tiende a aumentar a medida que se agranda la distancia al PNC $(H=42.1, p<0.001)$. El consumo de carne de tepezcuintle es diferente entre pueblos pero no se observa un patrón relacionado con la distancia al parque ( $\mathrm{H}$ $=18.3, \mathrm{p}<0.05)$ (Fig. 2).

El $80 \%$ de los entrevistados que conocen los chanchos cariblancos piensan que éstos viven en el parque y salen afuera al final de la época húmeda, y, en menor grado, al comienzo de la misma. La cacería de chanchos está concentrada en los momentos en que éstos salen del parque. Los tepezcuintles y los saínos son cazados 
indistintamente durante el año, generalmente en las mismas fincas de los pobladores. Los entrevistados más antiguos de la región hablaron de grupos de chanchos de entre 200 y 500 animales hace 20 años y de entre 80 y 200 actualmente.

El 50\% de la gente consigue la carne de monte regalada, el 26\% regalada y cazando, el 20\% exclusivamente cazando y el $4 \%$ la compra. El 25\% expresó un motivo económico para consumir carne de monte, el 30\% por la inaccesibilidad para comprar otras carnes y el $45 \%$ por gusto. La mayoría (65\%) de los entrevistados mayores de 50 años expresó haber cazado con frecuencia en su juventud, mientras que la mayoría (70\%) de los entrevistados jóvenes no caza. Las causas de la disminución de la cacería serían, en orden de importancia, las siguientes: la disminución de la abundancia de fauna, la reglamentación y la migración de gente joven hacia pueblos turísticos.

\section{DISCUSIÓN}

La carne de monte no es la principal fuente de proteínas en esta región ya que aún en los pueblos más inaccesibles el consumo de carne de monte se encuentra en quinto o cuarto lugar de consumo. Esto contrasta con lo encontrado en América del sur donde la carne de monte llega a constituir hasta el 90\% de la ingesta de proteínas (Redford y Robinson, 1987), y con algunas comunidades indígenas de centroamérica donde la carne de monte ocupa un importante lugar en la dieta (Carbonell, 1998; March, 1987; Nations, 1988; Nietschman, 1972). La diferencia se debe principalmente a que la cría para consumo de gallinas, pollos y cerdos es una práctica común en áreas rurales de la Península de Osa. Por otro lado, los pobladores de la Península de Osa probablemente no tienen la cacería tan arraigada culturalmente como los pobladores de la Amazonia o los grupos indígenas (Jorgenson, 1995; Meggars, 1971; Murphy y Murphy, 1985; entre otros).

En general los grupos rurales son menos selectivos que los grupos indígenas en cuanto a las especies silvestres que consumen (Ojasti, 1995). Sin embargo, grupos rurales en la Amazonía peruana consumen entre 15 y 30 especies de mamíferos. Indígenas Guaymíes de Punta Burica, sur de Costa Rica, consumen nueve especies de mamíferos (Carbonell, 1998). En este estudio se evidencia que los pobladores de la Península de Osa son altamente selectivos, ya que sólo consumen principalmente tres especies de mamíferos silvestres, y que no hay diferencia entre los indígenas Guaymíes de Osa y los campesinos en las especies consumidas. Las especies consumidas no son un reflejo de la disponibilidad de las mismas sino del aprecio que se tiene de su carne. Por ejemplo, en la zona abundan los pizotes y las guatuzas, ya que se acercan a los cultivos de maíz y yuca, pero su carne es en general despreciada. Por lo contrario, en algunos pueblos de la Amazonia la guatuza ocupa el segundo lugar de consumo según las tasas de cosecha (Redford y Robinson, 1987). Aparentemente, en la Península de Osa cuando la gente tiene la opción de consumir chancho de monte no 
consume saíno. Lo mismo se ha observado en otros sitios de América Latina donde ambas especies coexisten (Altrichter y Jiménez, 1999; Kiltie, 1980; Vickers, 1984). En la Península de Osa el saíno es consumido en aquellos pueblos donde la cacería de chanchos es rara o no existe (Fig. 2).

Se habla de cacería de subsistencia cuando un grupo asegura su alimentación por sus propios esfuerzos, a través de la explotación de su entorno (Nietschman, 1973). Por lo tanto, el objetivo directo de la cacería de subsistencia es la alimentación. Sin embargo, habría que considerar el aporte que la carne silvestre tiene en la dieta y los motivos para la cacería. Por ejemplo, los resultados de este estudio sugieren que la cacería de mamíferos silvestres en la Península es para autoconsumo pero no es estrictamente de subsistencia, ya que no es la principal fuente de carne, es altamente

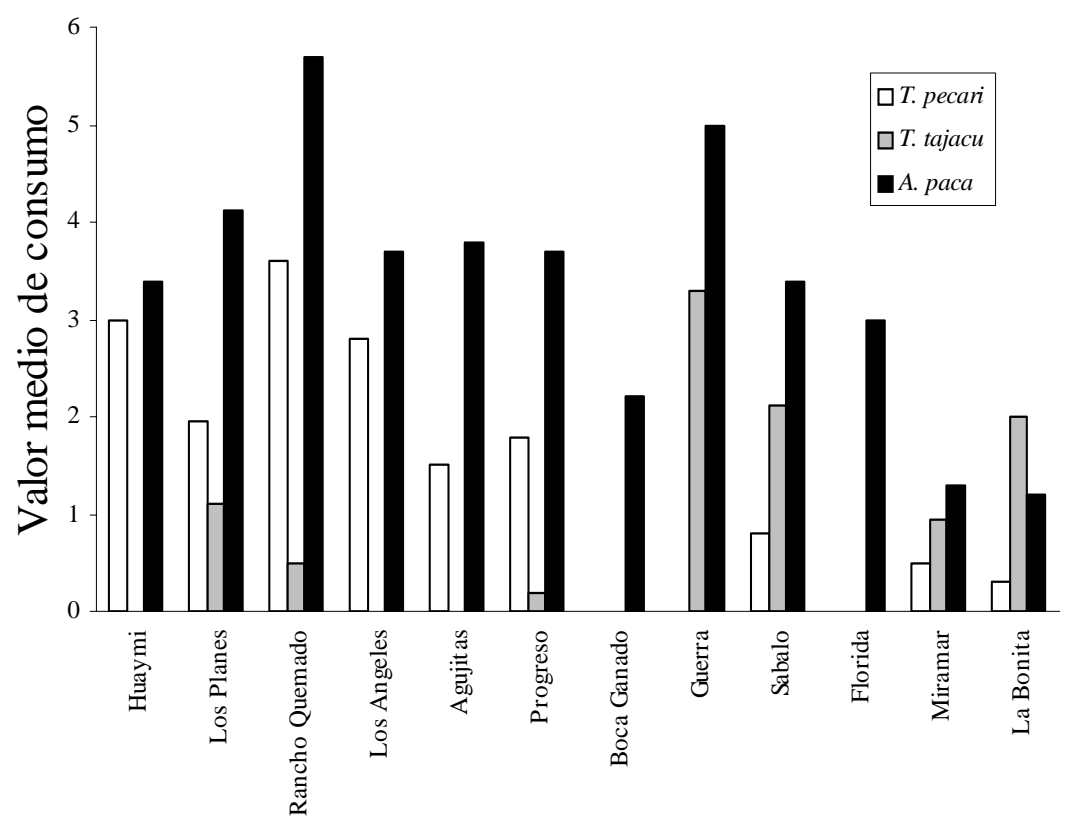

Figura 2. Valor medio de consumo de chanchos cara blanca, saínos y tepezcuintles en pueblos de la Península de Osa. Los pueblos están ordenados en forma descendente de acuerdo a la distancia del Parque Nacional Corcovado. Los tres primeros pueblos limitan con el parque. 
selectiva, y la carne de monte se consume en general más por su sabor que por motivos económicos.

Aparentemente, según comentarios de la gente, no hay poblaciones de chanchos viviendo en la península, fuera del PNC. Según la cantidad de bosque que queda en la península, es muy probable que ésto sea cierto, ya que no hay hábitat suficiente. El hecho de que exista mayor cacería de chanchos cerca del parque se debe probablemente a que éstos salen cerca de los límites en sus recorridos rutinarios, mientras que se desplazan por el resto de la península sólo una o dos veces al año. Sorprendentemente, los chanchos recorren largas distancias a través de la península llegando hasta la zona de Sábalo (Fig. 1). Este comportamiento ha sido observado también en la Amazonía Peruana, y se cree que está relacionado con los patrones de distribución de frutos (Bodmer, 1989; Bodmer, 1990; Kiltie y Terborgh, 1983; Sowls, 1984). La época en la que se observan más frecuentemente los chanchos desplazándose fuera del PNC coincide con el pico de lluvias y con una drástica disminución de la disponibilidad de frutos en el parque (Altrichter, 1997).

Aunque la cantidad de chanchos cazados parece baja y se evidencia una disminución de la actividad de cacería con relación a la época de colonización de la península, las poblaciones de chanchos también han disminuido (Vaughan, 1981), por lo que el efecto puede seguir siendo importante. Hace 30 años, la gente de la Península veía grupos de chanchos mucho más numerosos, y éstos llegaban hasta La Bonita, al norte de la Península (Fig. 1). Aparentemente, los chanchos que son cazados provienen del PNC, por lo que toda la población estaría susceptible a la cacería, y probablemente la protección del PNC no es suficiente.

Debido a la acelerada deforestación de la península en las últimas décadas, es difícil discernir el efecto de la cacería por separado. Esto indica que aunque la cacería de chanchos actualmente parece baja, puede haber afectado grandemente a las poblaciones de chanchos anteriormente y actualmente actuar con efecto sinérgico junto con la deforestación. Además, hay que considerar que este estudio sólo refleja la cacería realizada por pobladores de la península. Aparentemente, la cacería comercial por parte de gente de otras ciudades sigue siendo importante. Esto es un punto importante para continuar investigando ya que podría ser que exista una baja presión de cacería por parte de gente local pero que esté aumentando por parte de externos.

Considerando que para los pobladores locales una disminución del consumo de carne de chancho de monte no afectaría su alimentación, se debería intensificar el control de la cacería de chanchos en los pueblos más cercanos al PNC, especialmente durante el final de la época húmeda (octubre a diciembre). Por otro lado, el tepezcuintle puede ser una ayuda a la economía familiar y parece ser abundante en la península. Se deberían hacer estudios poblacionales de esta especie para revisar la posibilidad de otorgar permisos de cacería, dependiendo de la situación económica de la gente, o del lugar donde viven. Esto ayudaría a regular la cacería que de todos modos ocurre. 


\section{AGRADECIMIENTOS}

Agradezco a US Fish \& Wildlife Service y a la Unión Internacional para la Conservación de la Naturaleza por financiar este estudio. A R. Almeida y E. Baldiocea por su invaluable ayuda en el trabajo de campo. Al Programa Regional en Manejo de Vida Silvestre de la Universidad Nacional por su ayuda logística.

\section{LITERATURACITADA}

Altrichter, M. 1997. Estrategia de alimentación y comportamiento del chancho cariblanco Tayassu pecari en un bosque húmedo tropical de Costa Rica. Tesis de maestría. Universidad Nacional, Heredia, Costa Rica.

Altrichter, M e I. Jiménez. 1999. Caza y consumo de carne de monte en la comunidad de San Juan del Norte, Reserva Biológica Indio Maíz, Nicaragua. Boletín oficial de la Sociedad Mesamericana para la Biología y la Conservación, 4: 117-120.

Ander Egg, E. 1991. Técnicas de investigación social. El Ateneo. México, D.F. México.

Bodmer, R. E. 1989. Frugivory in Amazonian ungulates. Tesis doctoral. University of Cambridge, Cambridge, England.

Bodmer, R. E. 1990. Responses of ungulates to seasonal inundations in the Amazon Floodplain. Journal of Tropical Ecology, 6: 191-201.

Carbonell, F. 1998. Uso y abundancia de fauna en una comunidad indígena Guaymí en Costa Rica. Tesis de maestría. Universidad Nacional, Heredia, Costa Rica.

Jorgenson, J. P. 1995. Maya subsistence hunters in Quintana Roo, México. Oryx, 29: 49-57.

Kiltie, R. A. 1980. More on Amazon cultural ecology. Current Anthropology, 21: 541-546.

March, I. 1987. Los lacandones de México y su relación con los mamíferos silvestres: un estudio etnozoológico. Biótica, 12: 43-56.

Meggars, B. J. 1971. Amazonia, man and culture in a counterfeit paradise. Aldine-Atherton, Chicago and New York, EUA.

Murphy, Y. y R. F. Murphy. 1985. Women of the forest. Second edition., Columbia University Press, New York, EUA.

Nations, J. D. 1988. The Lacandón Maya. Pp. 86-88, en People of the tropical rain forest (J. S. Denslow y C. Padoch, eds.). University of California Press, Davis, California, EUA,

Nietschman, B. 1972. Hunting and fishing focus among the Miskito Indian of eastern Nicaragua. Human Ecology, 1: 41-67.

Nietschman, B. 1973. Between land and water: The subsistence ecology of the miskito indians, eastern Nicaragua. Seminar Press, New York, EUA.

Ojasti, J. 1993. Utilización de la fauna silvestre en América Latina. Situación y perspectivas para un manejo sostenible. Guía FAO: Conservación, Roma, Italia.

Ojasti, J. 1995. Uso y conservación de la fauna silvestre en la Amazonia. Tratado de Cooperación Amazonica. Secretaria Pro-Tempore, Perú.

Redford, K. H. y J. G. Robinson. 1987. The game of choice: patterns of Indian and colonist hunting in the neotropics. American Anthropologist, 89: 412-422. 
Robinson, J. G. y K. H. Redford (eds.). 1991. Neotropical wildlife use and conservation. The University of Chicago Press, Chicago, EUA.

Smith, N. J. 1976. Utilization of game along Brazil's Transamazon highway. Acta Amazonica, 6: 455-466.

Sowls, L. K. 1984. The peccaries. The University of Arizona Press, Tucson, Arizona, EUA.

Sowls, L. K. 1997. Javelinas and other Peccaries: Their biology, management and use. Second edition. The University of Arizona Press. Tucson, Arizona, EUA.

Vaughan, C. 1981. Parque Nacional Corcovado. Plan de Manejo y Desarrollo. Universidad Nacional. San José, Costa Rica.

Vickers, W. T. 1984. The faunal components of lowland South American hunting kills. Interciencia, 9: 366-376.

Vickers, W. T. 1991. Hunting yields and game composition over ten years in an Amazon Indian Territory. Pp. 53-81, en Neotropical Wildlife Use and Conservation (J. G. Robinson y K. H.. Redford, eds.). University of Chicago Press, Chicago, EUA. 